\title{
Instability of the Hocking-Stewartson pulse and its implications for three-dimensional Poiseuille flow
}

\author{
By Andrei L. Afendikov ${ }^{1}$ And Thomas J. Bridges ${ }^{2}$ \\ ${ }^{1}$ Keldysh Institute of Applied Mathematics, Miusskaya Square 4, Moscow, Russia \\ ${ }^{2}$ Department of Mathematics and Statistics, University of Surrey, \\ Guildford, Surrey GU2 $7 R Y, U K$
}

Received 12 June 2000; accepted 19 September 2000

The linear stability problem for the Hocking-Stewartson pulse, obtained by linearizing the complex Ginzburg-Landau (cGL) equation, is formulated in terms of the Evans function, a complex analytic function whose zeros correspond to stability exponents. A numerical algorithm based on the compound matrix method is developed for computing the Evans function. Using values in the cGL equation associated with spanwise modulation of plane Poiseuille flow, we show that the Hocking-Stewartson pulse associated with points along the neutral curve is always linearly unstable due to a real positive eigenvalue. Implications for the spanwise structure of nonlinear Poiseuille problem between parallel plates are also discussed.

Keywords: travelling wave; Evans function;

hydrodynamic stability; compound matrices

\section{Introduction}

Slow space and time modulation of the small-amplitude travelling periodic waves bifurcating from the neutral curve for two-dimensional plane Poiseuille flow leads to a complex Ginzburg-Landau model equation

$$
\frac{\partial A}{\partial \tau}=\gamma_{1} \frac{\partial^{2} A}{\partial X^{2}}+\gamma_{2} A+\gamma_{3}|A|^{2} A,
$$

where $\gamma_{1}, \gamma_{2}$ and $\gamma_{3}$ are complex constants which take explicit values at the nose of the neutral curve for Poiseuille flow (cf. Stewartson \& Stuart 1971). The spatial modulation in the derivation of (1.1) is in the streamwise direction. Note that modulation equation (1.1) has a reflection symmetry in the $X$-direction that is not a property of the original problem.

In the three-dimensional case, with two unbounded directions near the instability threshold, the Davey, Hocking and Stewartson (DHS) modulation system generalizes the one-dimensional equation (1.1) by including modulations in both the streamwise and spanwise directions (Davey et al. 1974). One can restrict the DHS system to one-dimensional problems by looking for particular classes of solutions. For instance, spatial modulation can be considered solely in the spanwise direction. 
Afendikov \& Mielke $(1995,2001 a)$ have recently reexamined this theory and shown that the form of the DHS system and the restricted equations depends crucially on what constraints are imposed on the spanwise and streamwise mass flux and the pressure gradient. When streamwise modulation is neglected, the spanwise modulation equations take the form

$$
\frac{\partial A}{\partial \tau}=b_{1} \frac{\partial^{2} A}{\partial Z^{2}}+b_{2} A+b_{3}|A|^{2} A+b_{4} \llbracket|A|^{2} \rrbracket A,
$$

where the complex coefficients depend on the mass fluxes and the pressure gradient and $\llbracket \cdot \rrbracket$ is an average which makes the equations non-local (cf. Afendikov \& Mielke $2001 b)$. For solutions which decay exponentially as $Z \rightarrow \pm \infty$, the non-local term vanishes and equations (1.2) reduce to the form (1.1), but with different values for the coefficients.

Numerical values for the coefficients in (1.2) for the case of zero mass flux in the spanwise direction and fixed pressure gradient in the streamwise direction have been reported for $\alpha \in\left(a_{1}, a_{2}\right)$ in Afendikov \& Mielke (2001a), where $\alpha$ is the streamwise wavenumber and $a_{1} \approx 0.98787, a_{2} \approx 1.0973$. The point $a_{1}$ represents the maximum value of $\alpha$ along the neutral curve, and $a_{2}$ represents the edge of the region where Squires' theorem is valid (see fig. 1 of Afendikov \& Mielke (2001a)).

All of these equations have an exact solution of the Hocking-Stewartson type

$$
A(Z, \tau)=\lambda L \mathrm{e}^{\mathrm{i} \nu \tau}(\operatorname{sech} \lambda Z)^{1+\mathrm{i} M},
$$

that is, even in $Z$ with $\lambda, L, \nu$ and $M$ satisfying specific constraints (cf. Hocking \& Stewartson 1972, p. 307). The important observation in Afendikov \& Mielke (2001a) is that the original hydrodynamical problem under the zero mean flux constraint is reflection symmetric in the spanwise direction, and hence it is possible to prove that pulse solutions of the reduced equation (1.2) actually persist as solutions of the threedimensional Poiseuille problem between parallel plates. These solutions are travelling waves, travelling downstream, and have a pulse structure in the spanwise direction. In parameter space near the Hocking-Stewartson (HS) pulse, Afendikov \& Mielke (1999, 2001a) have proved the existence of a countable family of multiple pulse solutions. The huge variety of these solutions suggests that they may play a role in the nonlinear transition to turbulence in three-dimensional Poiseuille flow. A natural question is whether pulse solutions of the Navier-Stokes problem of this type are stable. It is possible to reduce the hydrodynamic stability problem to the question of stability of the HS pulse solution of the complex Ginzburg-Landau (cGL) equation. But for the parameter regime of interest for hydrodynamics, even this reduced problem for hydrodynamics is not yet analytically tractable and hence should be tractable numerically. Since the spectral problem is stated on the real line, and it possesses non-trivial continuous spectrum, a naive numerical method fails.

In the original paper of Hocking \& Stewartson (1972), the initial-value problem for (1.1), with parameter values associated with streamwise modulation of plane Poiseuille flow, was studied numerically and, based on the numerics, they conjectured that the pulse solutions were unstable. The only study of the spectral problem associated with the linearization of (1.1) about the HS pulse is the work of Beyn \& Lorenz (1999), where for a particular range of parameters the spectrum is computed by restricting the spectral problem to a finite $Z$-interval and using collocation to reduce the spectral problem to a matrix eigenvalue problem. However, this approach 
requires the use of approximate boundary conditions for large $Z$ and the solution of the full spectral problem with large matrices. Therefore, some delicate properties of the spectrum can not be accurately recovered (see $\S 5$ ). Moreover, the parameter values studied are not relevant to spanwise modulation of the plane Poiseuille flow.

The purpose of this paper is threefold. Firstly, we formulate and study the linear stability of the HS pulse as a solution of the cGL equation using a numerical method that is based on a completely different idea. The eigenvalues of the linear stability problem are determined numerically, by developing an algorithm based on the compound matrix method for computing the Evans function associated with the linear stability of pulses. The method is tested with the use of several analytical properties of the spectrum showing excellent agreement with theoretical predictions. Secondly, we use numerical information to state some hypothesis on the particular eigenvalues and eigenfunctions of the problem. For instance, on a particular curve in parameter space, the stability exponent is found analytically. Finally, using calculated parameter values along the neutral curve, we determine the stability exponents of the HS pulse relevant to spanwise modulation of plane Poiseuille flow. Our numerical results indicate that the HS pulse solution for all parameter values along the neutral curve associated with spanwise modulation are linearly unstable. For the Poiseuille problem, this suggests that the pulse - and multi-pulse solutions obtained by Afendikov \& Mielke - bifurcate subcritically. Therefore, turning points in the branches of solutions could lead to the appearance of spatially complicated regimes for Reynolds numbers much lower than the instability threshold of the basic Poiseuille flow profile predicted by the Orr-Sommerfeld equation.

We take as a starting point the cGL equation in the scaled form

$$
\rho \mathrm{e}^{\mathrm{i} \psi} A_{t}=A_{x x}-(1+\mathrm{i} \omega)^{2} A+(1+\mathrm{i} \omega)(2+\mathrm{i} \omega)|A|^{2} A,
$$

where $A(x, t)$ is complex valued and $\rho>0, \omega, \psi$ are specified real parameters (precise values for these coefficients associated with the neutral curve are given in $\S 4$ ). Although we use the symbol $x$ for the spatial variable in (1.4), in the context of plane Poiseuille flow the $x$-variable is associated with spanwise modulation.

The HS pulse for this scaled equation takes the form

$$
A(x, t) \stackrel{\text { def }}{=} \hat{A}(x)=(\cosh x)^{-1-\mathrm{i} \omega} .
$$

The spectral problem is obtained by linearizing the real form of (1.4) about the HS pulse (1.5) and looking for solutions proportional to $\mathrm{e}^{\lambda t}$. Then the problem, with $\lambda \in \mathbb{C}$ as the spectral parameter, is formulated in an equivalent way as a system of the form

$$
\boldsymbol{v}_{x}=\boldsymbol{A}(x, \lambda) \boldsymbol{v}, \quad x \in \mathbb{R}, \quad \boldsymbol{v} \in \mathbb{C}^{4} .
$$

It is straightforward to show that the essential spectrum of problem (1.6) is given by

$$
S_{\text {ess }}=\left\{\rho^{-1} \mathrm{e}^{\mp \mathrm{i} \psi}\left(\omega^{2}-s^{2}-1\right) \mp 2 \mathrm{i} \rho^{-1} \omega \mathrm{e}^{\mp \mathrm{i} \psi}, s \in \mathbb{R}^{+}\right\},
$$

and hence we have the following necessary condition for stability of $S_{\text {ess }}$ :

$$
\cos \psi>0, \quad \cos \psi\left(\omega^{2}-1\right)-2 \omega \sin \psi<0 .
$$

Proc. R. Soc. Lond. A (2001) 
If this condition is violated, the trivial rest state is unstable and pulse solutions are marginally unstable due to the presence of the essential spectrum in the right half-plane. In the sequel it will be assumed that condition (1.8) is satisfied.

There are discrete eigenvalues as well. A discrete eigenvalue is a value of $\lambda \in \mathbb{C}$ for which (1.6) has a solution that decays exponentially as $x \rightarrow \pm \infty$. For instance, $\lambda=0$ is always an eigenvalue of multiplicity at least two, due to the rotation and translation symmetries of (1.4).

Equation (1.6) is in standard form for application of the theory of the Evans function. The Evans function is a complex analytic function whose zeros correspond to eigenvalues of the spectral problem associated with the linearization about a pulse solution (cf. Evans 1975; Alexander et al. 1990).

Numerical methods for computing the Evans function have been proposed by Evans \& Feroe (1977), Swinton \& Elgin (1990), and Pego et al. (1993). However, in all three cases, the 'systems at infinity' had only one eigenvalue with positive real part (see definition of systems at infinity in $\S 2$ ) that makes the computation of the Evans function straightforward. In the present case, the system at infinity has two eigenvalues with positive real part. These systems are stiff, and so standard numerical integrators for shooting will not work without some orthogonalization or other special treatment.

To compute the Evans function, we devise a numerical algorithm based on the compound matrix method. The compound matrix method is well known in hydrodynamic stability theory for integrating the Orr-Sommerfeld equation by shooting (cf. Ng \& Reid 1979, 1985; Davey 1979; Drazin \& Reid 1981). In fact, the compound matrix method is equivalent to integrating (1.6) restricted to $\bigwedge^{2}\left(\mathbb{C}^{4}\right)$ (Bridges 1999) and it eliminates the stiffness of equation (1.6). As far as we are aware, this is the first time that this method has been used to integrate the linear stability equations associated with pulses.

The algorithm is then used to compute the linear stability exponents for the state (1.5) for a range of parameters associated with plane Poiseuille flow. The implications for nonlinear three-dimensional flow between parallel plates are then discussed.

\section{Linear stability problem for the HS pulse}

Introduce new variables in order to write (1.4) in real coordinates,

$$
A=q_{1}+\mathrm{i} q_{2} \quad \text { and } \quad A_{x}=p_{1}+\mathrm{i} p_{2} .
$$

Then, using the matrices

$$
\boldsymbol{B}(\omega)=\left[\begin{array}{cc}
1 & -\omega \\
\omega & 1
\end{array}\right] \quad \text { and } \quad \boldsymbol{R}(\psi)=\left[\begin{array}{rr}
\cos \psi & -\sin \psi \\
\sin \psi & \cos \psi
\end{array}\right]
$$

equation (1.4) can be written as

$$
\left[\begin{array}{cc}
\mathbf{0} & \mathbf{0} \\
-\rho \boldsymbol{R}(\psi) & \mathbf{0}
\end{array}\right]\left(\begin{array}{l}
\boldsymbol{q} \\
\boldsymbol{p}
\end{array}\right)_{t}+\left(\begin{array}{l}
\boldsymbol{q} \\
\boldsymbol{p}
\end{array}\right)_{x}=\left[\begin{array}{c}
\boldsymbol{p} \\
\boldsymbol{B}(\omega)^{2} \boldsymbol{q}-\boldsymbol{B}(\omega)(\boldsymbol{I}+\boldsymbol{B}(\omega))\left(q_{1}^{1}+q_{2}^{2}\right) \boldsymbol{q}
\end{array}\right]
$$

or

$$
\boldsymbol{M} \boldsymbol{u}_{t}+\boldsymbol{u}_{x}=\boldsymbol{F}(\boldsymbol{u}), \quad \boldsymbol{u} \in \mathbb{R}^{4},
$$

Proc. R. Soc. Lond. A (2001) 
where $\boldsymbol{u}=(\boldsymbol{q}, \boldsymbol{p}) \in \mathbb{R}^{4}$. Note that the system (1.8) (or (2.2)) is a quasilinear parabolic system in the parameter domain of interest for hydrodynamical applications.

The basic state $\hat{\boldsymbol{z}}(x) \in \mathbb{R}^{4}$ satisfies

$$
\frac{\mathrm{d}}{\mathrm{d} x} \hat{\boldsymbol{z}}=\boldsymbol{F}(\hat{\boldsymbol{z}}) .
$$

Explicitly, the HS pulse (1.5) takes the form $\hat{\boldsymbol{z}}(x)=(\hat{\boldsymbol{q}}(x), \hat{\boldsymbol{p}}(x))$ with

$$
\hat{\boldsymbol{q}}(x)=\left(\begin{array}{c}
\operatorname{sech} x \cos (\omega \ln \cosh x) \\
-\operatorname{sech} x \sin (\omega \ln \cosh x)
\end{array}\right), \quad \hat{\boldsymbol{p}}(x)=\frac{\mathrm{d}}{\mathrm{d} x} \hat{\boldsymbol{q}}(x) .
$$

To formulate the linearized stability equation, let $\boldsymbol{u}(x, t)=\hat{\boldsymbol{z}}(x)+\hat{\boldsymbol{v}}(x, t)$, substitute into (2.2) and linearize about the basic state $\hat{\boldsymbol{z}}(x)$,

$$
\boldsymbol{M} \hat{\boldsymbol{v}}_{t}+\hat{\boldsymbol{v}}_{x}=D \boldsymbol{F}(\hat{\boldsymbol{z}}(x)) \hat{\boldsymbol{v}}, \quad \text { where } D \boldsymbol{F}(\hat{\boldsymbol{z}}(x))=\left[\begin{array}{cc}
\mathbf{0} & \boldsymbol{I} \\
\boldsymbol{P}(x) & \mathbf{0}
\end{array}\right]
$$

and

$$
\boldsymbol{P}(x)=\boldsymbol{B}(\omega)^{2}-\boldsymbol{B}(\omega)(\boldsymbol{I}+\boldsymbol{B}(\omega))\left(|\hat{\boldsymbol{q}}|^{2} \boldsymbol{I}+2 \hat{\boldsymbol{q}} \hat{\boldsymbol{q}}^{\mathrm{T}}\right) .
$$

Introduce a spectral ansatz: $\hat{\boldsymbol{v}}(x, t)=\boldsymbol{v}(x, \lambda) \mathrm{e}^{\lambda t}$, then $(2.4)$ reduces to

$$
\boldsymbol{v}_{x}=\boldsymbol{A}(x, \lambda) \boldsymbol{v}, \quad \boldsymbol{v} \in \mathbb{C}^{4},
$$

with

$$
\boldsymbol{A}(x, \lambda)=\left[\begin{array}{cc}
\mathbf{0} & \boldsymbol{I} \\
\boldsymbol{P}(x)+\lambda \rho \boldsymbol{R}(\psi) & \mathbf{0}
\end{array}\right]
$$

It is apparent from (2.7) that the matrix $\boldsymbol{A}(x, \lambda)$ has the following useful property:

$$
\operatorname{Tr}(\boldsymbol{A}(x, \lambda))=0,
$$

independent of $x$ and $\lambda$.

Equation (2.6) is the main equation that we will study to determine the linear stability properties of the HS pulse.

Definition 2.1. The HS pulse is said to be linearly unstable or spectrally unstable if, for some fixed $\lambda \in \mathbb{C}$ with $\operatorname{Re}(\lambda)>0$, there exists a solution of (2.6) which decays exponentially as $x \rightarrow \pm \infty$.

The nonlinear stability will not be considered; however, see Alexander et al. (1990) for a discussion of how spectral information relates to nonlinear stability for parabolic partial differential equations.

\section{The Evans function}

The system (2.6) is in standard form to apply the Evans function formulation.

It follows from (2.6), (2.7) and the property that $|\hat{\boldsymbol{q}}| \rightarrow 0$ as $x \rightarrow \pm \infty$, that the limit as $x \rightarrow \pm \infty$ of $\boldsymbol{A}(x, \lambda)$ exists. Define

$$
\boldsymbol{A}_{\infty}(\lambda) \stackrel{\text { def }}{=} \lim _{x \rightarrow \pm \infty} \boldsymbol{A}(x, \lambda)=\left[\begin{array}{cc}
\mathbf{0} & \boldsymbol{I} \\
\boldsymbol{B}(\omega)^{2}+\lambda \rho \boldsymbol{R}(\psi) & \mathbf{0}
\end{array}\right]
$$


With reasonable restrictions on the values of $\omega, \rho$ and $\psi$, we will show below that for all $\lambda \in \mathbb{C}$ with $\operatorname{Re}(\lambda)>0$, the spectrum of $\boldsymbol{A}_{\infty}(\lambda)$ has exactly two eigenvalues $\mu_{1,2}(\lambda)$ with positive real part and two $\mu_{3,4}(\lambda)$ with negative real part with the corresponding eigenvectors $\xi_{j}, j=1, \ldots, 4$.

From standard results of the asymptotic theory for linear ordinary differential equations (ODEs), it follows that there are two linear independent solutions $v_{j}(x, \lambda)$, $j=1,2$, to system (2.6) that correspond to the unstable subspace of $\boldsymbol{A}_{\infty}(\lambda)$ and which satisfy the asymptotic estimates

$$
\lim _{x \rightarrow-\infty} \mathrm{e}^{-\mu_{j}(\lambda) x} v_{j}(x, \lambda)=\xi_{j}, \quad j=1,2 .
$$

Similarly, there are two solutions $v_{j}(x, \lambda), j=3,4$, that correspond to the stable subspace of $\boldsymbol{A}_{\infty}(\lambda)$ and which satisfy

$$
\lim _{x \rightarrow \infty} \mathrm{e}^{-\mu_{j}(\lambda) x} v_{j}(x, \lambda)=\xi_{j}, \quad j=3,4 .
$$

If, for some $\lambda \in \mathbb{C}$, these two spaces have a non-trivial intersection, then $\lambda$ will be an eigenvalue. The function, $D(\lambda)$, which measures whether these two spaces intersect is called the Evans function and it is essentially a Wronskian evaluated on the vectors $v_{j}, j=1, \ldots, 4$ (see Evans 1975; Alexander et al. 1990).

(a) The system at infinity, $\boldsymbol{A}_{\infty}(\lambda)$

Define $\Delta(\mu, \lambda)=\operatorname{det}\left[\mu \boldsymbol{I}-\boldsymbol{A}_{\infty}(\lambda)\right]$, then

$$
\Delta(\mu, \lambda)=\operatorname{det}\left[\mu^{2} \boldsymbol{I}-\boldsymbol{B}(\omega)^{2}-\lambda \rho \boldsymbol{R}(\psi)\right]=\mu^{4}-2 \tau(\lambda) \mu^{2}+\delta(\lambda),
$$

where

$$
\tau(\lambda)=\frac{1}{2} \operatorname{Tr}\left(\boldsymbol{B}(\omega)^{2}+\lambda \rho \boldsymbol{R}(\psi)\right) \quad \text { and } \quad \delta(\lambda)=\operatorname{det}\left[\boldsymbol{B}(\omega)^{2}+\lambda \rho \boldsymbol{R}(\psi)\right] .
$$

Explicitly,

$$
\boldsymbol{B}(\omega)^{2}+\lambda \rho \boldsymbol{R}(\psi)=\left[\begin{array}{cc}
1-\omega^{2}+\lambda \rho \cos \psi & -2 \omega-\lambda \rho \sin \psi \\
2 \omega+\lambda \rho \sin \psi & 1-\omega^{2}+\lambda \rho \cos \psi
\end{array}\right]
$$

and so

$$
\tau(\lambda)=1-\omega^{2}+\lambda \rho \cos \psi \quad \text { and } \quad \delta(\lambda)=\left(1-\omega^{2}+\lambda \rho \cos \psi\right)^{2}+(2 \omega+\lambda \rho \sin \psi)^{2} .
$$

The four eigenvalues of the matrix $\boldsymbol{A}_{\infty}(\lambda)$, the $\mu$-roots of $\Delta(\mu, \lambda)=0$, are determined from the relation

$$
\mu^{2}=(1 \pm \mathrm{i} \omega)^{2}+\lambda \rho \mathrm{e}^{ \pm \mathrm{i} \psi} .
$$

The two eigenvalues with positive real part are given explicitly by

$$
\left[(1-\mathrm{i} \omega)^{2}+\lambda \rho \mathrm{e}^{-\mathrm{i} \psi}\right]^{1 / 2} \text { and }\left[(1+\mathrm{i} \omega)^{2}+\lambda \rho \mathrm{e}^{+\mathrm{i} \psi}\right]^{1 / 2},
$$

with the positive square root taken in both cases.

When $\rho \sin \psi \neq 0$, there is a single point $\lambda_{0}$ in the complex $\lambda$-plane where $\boldsymbol{A}_{\infty}\left(\lambda_{0}\right)$ has a double root. The discriminant of $\Delta(\mu, \lambda)$ is

$$
\mathrm{d}(\lambda)=-(2 \omega+\lambda \rho \sin \psi)^{2},
$$

Proc. R. Soc. Lond. A (2001) 
and it vanishes when

$$
\lambda=\lambda_{0}=\frac{-2 \omega}{\rho \sin \psi} .
$$

At $\lambda=\lambda_{0}$, the double roots are

$$
\mu_{0}^{2}=\tau\left(\lambda_{0}\right)=1-\omega^{2}+\lambda_{0} \rho \cos \psi=1-\omega^{2}-2 \omega \cot \psi
$$

Since the marginal stability condition (1.8) is assumed to be satisfied, it follows that $\tau\left(\lambda_{0}\right)<0$ and $\lambda_{0} \in S_{\text {ess }}$. In the essential spectrum, $S_{\text {ess }}$, the classically defined Evans function, is not in general analytic. However, recent results have shown how to extend the Evans function so that it is defined in the essential spectrum as well (cf. Gardner \& Zumbrun 1998; Kapitula \& Sandstede 1998). Since we assume the essential spectrum is stable, we will restrict attention in the numerical computations to the right-half $\lambda$-plane, where only discrete eigenvalues are possible and the classical Evans function is well defined.

\section{(b) The spectral ODE on $\bigwedge^{2}\left(\mathbb{C}^{4}\right)$}

With the eigenvalue structure of $\boldsymbol{A}_{\infty}(\lambda)$, the system (2.6) is in standard form for application of the Evans function theory. Define $\bigwedge^{2}\left(\mathbb{C}^{4}\right)$ to be the set of all two forms on $\mathbb{C}^{4}$. Let $\boldsymbol{U}^{+}(x, \lambda) \in \bigwedge^{2}\left(\mathbb{C}^{4}\right)$ represent the two-dimensional space of solutions which are bounded as $x \rightarrow+\infty$, and let $\boldsymbol{U}^{-}(x, \lambda) \in \bigwedge^{2}\left(\mathbb{C}^{4}\right)$ represent the two-dimensional space of solutions which are bounded as $x \rightarrow-\infty$. The Evans function is then defined by

$$
\tilde{D}(\lambda)=\exp \left[-\int_{0}^{x} \operatorname{Tr}(\boldsymbol{A}(s, \lambda)) \mathrm{d} s\right] \boldsymbol{U}^{+}(x, \lambda) \wedge \boldsymbol{U}^{-}(x, \lambda) \quad \forall \lambda \in \Lambda,
$$

where $\Lambda$ is taken to be the right-half complex plane. The Evans function defined in this way is independent of $x$, a complex analytic function with values in $\Lambda^{4}\left(\mathbb{C}^{4}\right)$, and values of $\lambda \in \Lambda$ satisfying $\tilde{D}(\lambda)=0$ are unstable eigenvalues (cf. Alexander et al. 1990). Expression (3.3) can be simplified and put into a form suitable for numerical computation.

Proposition 3.1. The Evans function $\tilde{D}(\lambda)$ is proportional to the standard volume form $\mathcal{V}$ in $\mathbb{C}^{4}$,

$$
\tilde{D}(\lambda)=D(\lambda) \mathcal{V}
$$

where

$$
D(\lambda)=\left[\boldsymbol{U}^{+}(x, \lambda), \Sigma \overline{\boldsymbol{U}^{-}(x, \lambda)}\right] .
$$

The pairing $[\cdot, \cdot]$ is a complex inner product on $\mathbb{C}^{6}$ with conjugation of the second entry, and

$$
\Sigma=\left(\begin{array}{cccccc}
0 & 0 & 0 & 0 & 0 & 1 \\
0 & 0 & 0 & 0 & -1 & 0 \\
0 & 0 & 0 & 1 & 0 & 0 \\
0 & 0 & 1 & 0 & 0 & 0 \\
0 & -1 & 0 & 0 & 0 & 0 \\
1 & 0 & 0 & 0 & 0 & 0
\end{array}\right)
$$

Proc. R. Soc. Lond. A (2001) 
Proof. Since the trace of $\boldsymbol{A}(x, \lambda)$ vanishes (see (2.8)), the exponential factor is unity. The functions $\boldsymbol{U}^{ \pm}$are two forms, and so the right-hand side of (3.3) is an element of $\bigwedge^{4}\left(\mathbb{C}^{4}\right)$.

Take $\boldsymbol{e}_{1}, \ldots, \boldsymbol{e}_{4}$ to be an orthonormal basis for $\mathbb{C}^{4}$ and choose $\mathcal{V}=\boldsymbol{e}_{1} \wedge \boldsymbol{e}_{2} \wedge \boldsymbol{e}_{3} \wedge \boldsymbol{e}_{4}$ to be the volume form. Then

$$
\tilde{D}(\lambda)=\boldsymbol{U}^{+}(x, \lambda) \wedge \boldsymbol{U}^{-}(x, \lambda)=D(\lambda) \mathcal{V} .
$$

An explicit expression for $D(\lambda)$ (which will be used for the numerics) can be obtained by introducing a natural basis for $\bigwedge^{2}\left(\mathbb{C}^{4}\right)$,

$$
\left.\begin{array}{lll}
\omega_{1}=e_{1} \wedge \boldsymbol{e}_{2}, & \omega_{2}=e_{1} \wedge \boldsymbol{e}_{3}, & \omega_{3}=\boldsymbol{e}_{1} \wedge \boldsymbol{e}_{4}, \\
\omega_{4}=e_{2} \wedge \boldsymbol{e}_{3}, & \omega_{5}=e_{2} \wedge \boldsymbol{e}_{4}, & \omega_{6}=\boldsymbol{e}_{3} \wedge \boldsymbol{e}_{4}
\end{array}\right\}
$$

Expand $\boldsymbol{U}^{ \pm}(x, \lambda)$ in terms of this basis,

$$
\boldsymbol{U}^{ \pm}(x, \lambda)=\sum_{j=1}^{6} U_{j}^{ \pm}(x, \lambda) \omega_{j} .
$$

Substitution of these expressions into (3.5) leads to

$$
\begin{aligned}
\boldsymbol{U}^{+}(x, \lambda) \wedge \boldsymbol{U}^{-}(x, \lambda) & =\sum_{i=1}^{6} \sum_{j=1}^{6} U_{j}^{+}(x, \lambda) U_{j}^{-}(x, \lambda) \omega_{i} \wedge \omega_{j}, \\
& =\left[\boldsymbol{U}^{+}(x, \lambda), \Sigma \overline{\boldsymbol{U}^{-}(x, \lambda)}\right] \mathcal{V},
\end{aligned}
$$

which is the required expression.

The matrix $\Sigma$ is in fact a representation of the Hodge star operator (cf. Bridges \& Derks 1999), and general aspects of the numerics of exterior algebra which lie behind these constructions are given in Allen \& Bridges (2000).

The function $D(\lambda)$ will be computed numerically using the compound matrix method, where the components of the vector $\boldsymbol{U}^{ \pm}(x, \lambda)$ are the six coordinates relative to the basis (3.6). We will construct differential equations for $\boldsymbol{U}^{ \pm}(x, \lambda)$ by restricting $(2.6)$ to $\bigwedge^{2}\left(\mathbb{C}^{4}\right)$.

The functions $\boldsymbol{U}^{ \pm}(x, \lambda)$ satisfy the differential equation

$$
\frac{\mathrm{d}}{\mathrm{d} x} \boldsymbol{U}^{ \pm}(x, \lambda)=\boldsymbol{A}^{(2)}(x, \lambda) \boldsymbol{U}^{ \pm}(x, \lambda),
$$

where $\boldsymbol{A}^{(2)}(x, \lambda)$ is a $6 \times 6$ matrix which is obtained by restricting $\boldsymbol{A}(x, \lambda)$ to $\bigwedge^{2}\left(\mathbb{C}^{4}\right)$. An explicit algorithm for doing this is given in the appendix of Bridges (1999).

For the system $(2.6), \boldsymbol{A}^{(2)}(x, \lambda)$ can be easily constructed. Write

$$
\boldsymbol{A}(x, \lambda)=\left[\begin{array}{cccc}
0 & 0 & 1 & 0 \\
0 & 0 & 0 & 1 \\
a_{31} & a_{32} & 0 & 0 \\
a_{41} & a_{42} & 0 & 0
\end{array}\right]
$$

where

$$
\begin{aligned}
\left(\begin{array}{ll}
a_{31} & a_{32} \\
a_{41} & a_{42}
\end{array}\right) & =\boldsymbol{P}(x)+\lambda \rho \boldsymbol{R}(\psi) \\
& =\boldsymbol{B}(\omega)^{2}-\boldsymbol{B}(\omega)[\boldsymbol{I}+\boldsymbol{B}(\omega)]\left(|\hat{\boldsymbol{u}}|^{2} \boldsymbol{I}+2 \hat{\boldsymbol{u}} \hat{\boldsymbol{u}}^{\mathrm{T}}\right)+\lambda \rho \boldsymbol{R}(\psi),
\end{aligned}
$$

Proc. R. Soc. Lond. A (2001) 
and so

$$
\begin{aligned}
& a_{31}=\lambda \rho \cos \psi+1-\omega^{2}-\left(2-\omega^{2}\right)\left(\hat{q}_{2}^{2}+3 \hat{q}_{1}^{2}\right)+6 \omega \hat{q}_{1} \hat{q}_{2}, \\
& a_{32}=-\lambda \rho \sin \psi-2 \omega-2\left(2-\omega^{2}\right) \hat{q}_{1} \hat{q}_{2}+3 \omega\left(\hat{q}_{1}^{2}+3 \hat{q}_{2}^{2}\right), \\
& a_{41}=\lambda \rho \sin \psi+2 \omega-2\left(2-\omega^{2}\right) \hat{q}_{1} \hat{q}_{2}-3 \omega\left(3 \hat{q}_{1}^{2}+\hat{q}_{2}^{2}\right), \\
& a_{42}=\lambda \rho \cos \psi+1-\omega^{2}-\left(2-\omega^{2}\right)\left(\hat{q}_{1}^{2}+3 \hat{q}_{2}^{2}\right)-6 \omega \hat{q}_{1} \hat{q}_{2} .
\end{aligned}
$$

The induced matrix on $\bigwedge^{2}\left(\mathbb{C}^{4}\right)$ is then

$$
\boldsymbol{A}^{(2)}(x, \lambda)=\left[\begin{array}{cccccc}
0 & 0 & 1 & -1 & 0 & 0 \\
a_{32} & 0 & 0 & 0 & 0 & 0 \\
a_{42} & 0 & 0 & 0 & 0 & 1 \\
-a_{31} & 0 & 0 & 0 & 0 & -1 \\
-a_{41} & 0 & 0 & 0 & 0 & 0 \\
0 & -a_{41} & a_{31} & -a_{42} & a_{32} & 0
\end{array}\right]
$$

(see Bridges 1999). This completes the specification of the systems (3.8). It remains to specify the starting vectors for the systems (3.8).

Define

$$
\boldsymbol{A}_{\infty}^{(2)}(\lambda)=\lim _{x \rightarrow \pm \infty} \boldsymbol{A}^{(2)}(x, \lambda)
$$

then

$$
\boldsymbol{A}_{\infty}^{(2)}(\lambda)=\left[\begin{array}{cccccc}
0 & 0 & 1 & -1 & 0 & 0 \\
-p(\lambda) & 0 & 0 & 0 & 0 & 0 \\
\tau(\lambda) & 0 & 0 & 0 & 0 & 1 \\
-\tau(\lambda) & 0 & 0 & 0 & 0 & -1 \\
-p(\lambda) & 0 & 0 & 0 & 0 & 0 \\
0 & -p(\lambda) & \tau(\lambda) & -\tau(\lambda) & -p(\lambda) & 0
\end{array}\right] .
$$

The starting vectors for the systems (3.8) are the eigenvectors of $\boldsymbol{A}_{\infty}^{(2)}(\lambda)$ associated with the eigenvalues of $\boldsymbol{A}_{\infty}^{(2)}(\lambda)$ of largest positive and largest negative real part.

The characteristic polynomial of $\boldsymbol{A}_{\infty}^{(2)}(\lambda)$ is

$$
\operatorname{det}\left[\boldsymbol{A}_{\infty}^{(2)}(\lambda)-\sigma \boldsymbol{I}_{6}\right]=\sigma^{2}\left(\sigma^{4}-4 \tau(\lambda) \sigma^{2}-4 p(\lambda)^{2}\right),
$$

where $p(\lambda)=2 \omega+\lambda \rho \sin \psi$. Therefore, the non-zero $\sigma$-roots are determined from

$$
\sigma^{2}=2 \tau(\lambda) \pm 2 \sqrt{\tau^{2}+p^{2}} .
$$

The root with largest positive real part is

$$
\sigma^{-}(\lambda)=\sqrt{2} \sqrt{\tau+\sqrt{\tau^{2}+p^{2}}} .
$$

This root is in fact the sum of the two roots of $\boldsymbol{A}_{\infty}(\lambda)$ with positive real part. The root with largest negative real part, $\sigma^{+}(\lambda)$, is the negative of (3.11).

The eigenvectors of $\boldsymbol{A}_{\infty}^{(2)}(\lambda)$ satisfy

$$
\boldsymbol{A}_{\infty}^{(2)}(\lambda) \xi=\sigma \xi, \quad \xi \in \mathbb{C}^{6} .
$$

Proc. R. Soc. Lond. A (2001) 
A straightforward calculation shows that when $\sigma \neq 0$,

$$
\xi(\lambda)=\mathbb{C}\left(\begin{array}{c}
2 \sigma \\
-2 p \\
\sigma^{2} \\
-\sigma^{2} \\
-2 p \\
\sigma\left(\sigma^{2}-2 \tau\right)
\end{array}\right),
$$

where $\mathbb{C}$ represents an arbitrary complex constant. Therefore, the eigenvectors corresponding to $\sigma^{ \pm}(\lambda)$ are given by $\xi^{ \pm}(\lambda)$, with $\sigma$ replaced in (3.12) by $\sigma^{ \pm}(\lambda)$.

\section{The numerical algorithm}

The infinite $x$-domain is truncated to $-L_{\infty}<x<L_{\infty}$ with $L_{\infty}$ suitably chosen. Then the main part of the algorithm involves numerical integration of the following two systems with $\lambda$ fixed:

$$
\frac{\mathrm{d}}{\mathrm{d} x} \boldsymbol{U}^{+}=\boldsymbol{A}^{(2)}(x, \lambda) \boldsymbol{U}^{+},\left.\quad \boldsymbol{U}^{+}(x, \lambda)\right|_{x=L_{\infty}}=\xi^{+}(\lambda) \text { for } L_{\infty}>x>0
$$

and

$$
\frac{\mathrm{d}}{\mathrm{d} x} \boldsymbol{U}^{-}=\boldsymbol{A}^{(2)}(x, \lambda) \boldsymbol{U}^{-},\left.\quad \boldsymbol{U}^{-}(x, \lambda)\right|_{x=-L_{\infty}}=\xi^{-}(\lambda) \quad \text { for }-L_{\infty}<x<0 .
$$

These two systems are integrated using the second-order implicit Gauss-Legendre Runge-Kutta (GLRK) method (higher-order GLRK methods could also have been easily used but did not appear to be necessary). Writing the above systems as $\boldsymbol{U}_{x}=$ $\boldsymbol{B}(x) \boldsymbol{U}$, the GLRK algorithm takes the form

$$
\boldsymbol{U}^{n+1}=\boldsymbol{U}^{n}+\Delta x \boldsymbol{B}_{n+1 / 2} \boldsymbol{U}^{n+1 / 2},
$$

where $\boldsymbol{B}_{n+1 / 2}=\boldsymbol{B}\left(x_{n+1 / 2}\right)$ and $\boldsymbol{U}^{n+1 / 2}=\frac{1}{2}\left(\boldsymbol{U}^{n}+\boldsymbol{U}^{n+1}\right)$. Therefore, one step of the integration is given by

$$
\boldsymbol{U}^{n+1}=\left[\boldsymbol{I}-\frac{1}{2} \Delta x \boldsymbol{B}_{n+1 / 2}\right]^{-1}\left[\boldsymbol{I}+\frac{1}{2} \Delta x \boldsymbol{B}_{n+1 / 2}\right] \boldsymbol{U}^{n} .
$$

At $x=0$, the solutions of (4.1) and (4.2) are combined to give a numerical approximation to the Evans function,

$$
D(\lambda)=\left[\boldsymbol{U}^{+}(0, \lambda), \Sigma \overline{\boldsymbol{U}^{-}(0, \lambda)}\right]+\varepsilon\left(L_{\infty}, \Delta x\right),
$$

where $\varepsilon\left(L_{\infty}, \Delta x\right)$ is the error due to the numerical approximation.

\section{Numerical tests and accuracy}

The case $\omega=\psi=0$ provides a good check for the algorithm because the spectrum of the linearized system can be computed explicitly. In this case, the system (2.6) decouples into two second-order systems:

$$
\frac{\mathrm{d}^{2} v_{1}}{\mathrm{~d} x^{2}}+\left(6 \hat{q}_{1}(x)^{2}-1\right) v_{1}=\lambda v_{1} \quad \text { and } \quad \frac{\mathrm{d}^{2} v_{2}}{\mathrm{~d} x^{2}}+\left(2 \hat{q}_{1}(x)^{2}-1\right) v_{2}=\lambda v_{2},
$$

Proc. R. Soc. Lond. A (2001) 
Table 1. Computed unstable eigenvalue when $\psi=\omega=0, \rho=1$

\begin{tabular}{rlll}
\hline$L_{\infty}$ & \multicolumn{1}{c}{$\Delta x$} & computed $\lambda$ \\
\hline 5.0 & 0.01 & 3.00005333264 \\
5.0 & 0.001 & 3.00000053332 \\
5.0 & 0.0001 & 3.00000000532 \\
10.0 & 0.01 & 3.00005333266 \\
10.0 & 0.001 & 3.00000053333 \\
10.0 & 0.0001 & 3.00000000533 \\
\hline
\end{tabular}

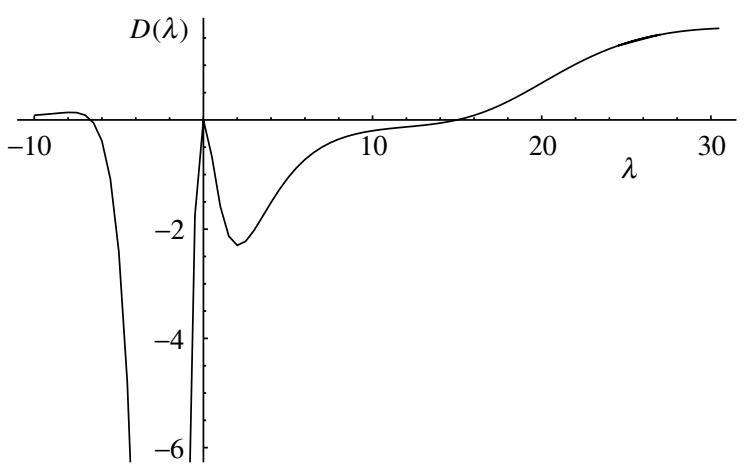

Figure 1. $D(\lambda)$ versus $\lambda$ along the real axis for $\omega=3, \psi=\tan ^{-1}(2), \rho=1 / \sqrt{ } 5$.

with $\hat{q}_{1}(x)=\operatorname{sech}(x)$. The spectrum of these two equations can be computed explicitly, by converting them to associated Legendre equations. The first equation has exactly two discrete eigenvalues at $\lambda=0$ and $\lambda=3$, and the second equation has only $\lambda=0$ as a discrete eigenvalue. The eigenfunction associated with the eigenvalue $\lambda=3$ is $v_{1}(x)=\operatorname{sech}^{2}(x)$.

The computed value of the unstable eigenvalue is shown in table 1 as a function of $L_{\infty}$ and $\Delta x$. Even though the numerical method used to integrate the ODEs was only second-order accurate, the computed results are very good. Note also the rapid convergence of the results with increasing $L_{\infty}$. The difference between the computed eigenvalue for $L_{\infty}=5$ and $L_{\infty}=10$ is insignificant, but that is not surprising since the eigenfunction corresponding to this eigenvalue decays as $x \rightarrow \pm \infty$ twice as fast as $\hat{q}_{1}(x)$.

The values of $\lambda$ in table 1 were computed by first plotting $D(\lambda)$ in (4.4) to get an approximation to the unstable eigenvalue, and then Newton's method was used to refine the roots of $D(\lambda)$.

In fig. 11 of Beyn \& Lorenz (1999), the spectrum is shown for the linearization about the HS pulse with $\omega=3, \rho=1 / \sqrt{ } 5$ and $\psi=\tan ^{-1}(2)$. They find exactly three discrete eigenvalues: a double root at $\lambda=0$ and simple roots at $\lambda \approx-7.0$ and $\lambda \approx+15.0$. Since all three of these roots are on the real axis, we plotted the Evans function numerically along the real axis for these parameter values and the result is shown in figure 1. There is excellent qualitative agreement with fig. 11 of Beyn \& Lorenz. The plot of $D(\lambda)$ shows precisely a double root at $\lambda=0$ and simple roots at approximately -6.6357 and +15 . The Evans function on figure 1 has large amplitude and a part of the graph is omitted, since $D(-2) \approx-32$.

Proc. R. Soc. Lond. A (2001) 
Table 2. Computed unstable eigenvalue when $\psi=\tan ^{-1}(2), \omega=3, \rho=1 / \sqrt{ } 5$

\begin{tabular}{llll}
\hline$L_{\infty}$ & \multicolumn{1}{c}{$\Delta x$} & \multicolumn{1}{c}{ computed $\lambda$} \\
\hline 15.0 & 0.003 & 15.0001280585 \\
15.0 & 0.00075 & 15.0000319595 \\
15.0 & 0.0001 & 15.0000005681 \\
15.0 & 0.00001 & 15.0000000056 \\
30.0 & 0.003 & 15.0005113572 \\
30.0 & 0.0001 & 15.0000005681 \\
\hline
\end{tabular}

Computed values of the positive root as a function of $L_{\infty}$ and $\Delta x$ are shown in table 2 .

Our calculations demonstrate a rather complicated dependence of the discrete spectrum of problem $(2.6)$ on parameters $(\psi, \omega)$ and there is little hope of finding the eigenvalue with the maximal real part, and its corresponding eigenfunction analytically. Nevertheless, symmetries of the cGL problem may help to find some of the eigenfunctions explicitly. For example, the scaling invariance of the cGL problem can be used to give an explicit expression for the generalized eigenfunction which exists at $\lambda=0$ when

$$
\operatorname{Re}\left(\mathrm{e}^{-\mathrm{i} \psi}(1+\mathrm{i} \omega)^{2}\right)=0 .
$$

To demonstrate this, note that the equation

$$
\rho \mathrm{e}^{\mathrm{i} \psi} A_{t}=A_{x x}-\mu(1+\mathrm{i} \omega)^{2} A+(1+\mathrm{i} \omega)(2+\mathrm{i} \omega)|A|^{2} A, \quad \mu>0,
$$

has the family of stationary solutions

$$
H(\mu, x) \stackrel{\text { def }}{=} \sqrt{\mu} \hat{A}(\sqrt{\mu} x) .
$$

Differentiating (5.3), evaluated at the solution (5.4), with respect to $\mu$ and setting $\mu=1$ results in

$$
-\mathrm{e}^{\mathrm{i} \psi}(1+\mathrm{i} \omega)^{2} \hat{A}=B_{x x}-(1+\mathrm{i} \omega)^{2} B+(1+\mathrm{i} \omega)(2+\mathrm{i} \omega)\left(2|\hat{A}|^{2} B+\hat{A}^{2} \bar{B}\right),
$$

with $B(x)=H_{\mu}(1, x)$. Hence $B(x)$ is the generalized eigenfunction to the eigenvalue $\lambda=0$ if (5.2) is satisfied. The existence of this generalized eigenfunction was first shown by Mielke (2000) using a different argument.

When the above condition is satisfied, the Evans function has at least a triple root at $\lambda=0$. This triple root is delicate to capture using a matrix method and Beyn \& Lorenz (1999) were unable to recover it numerically. In figure 2, a plot of $D(\lambda)$ versus $\lambda$ along the real axis is shown, for parameter values associated with a triple root.

In the present case, the numerical evidence shows that the computed Evans function $D(\lambda)$ captures quite clearly the algebraic property that $\lambda=0$ is a triple root. To compare with the numerical results of Beyn \& Lorenz (1999), we take $L_{\infty}=15$, $\Delta x=0.0001$ and get

$$
D^{\prime}(0) \approx-1.6 \times 10^{-7}, \quad D^{\prime \prime}(0) \approx 5.2 \times 10^{-7}, \quad D^{\prime \prime \prime}(0) \approx-0.018 .
$$

To within numerical accuracy, these results indicate that $\lambda=0$ has algebraic multiplicity 3. Qualitatively, the result is rather good considering that the numerical 


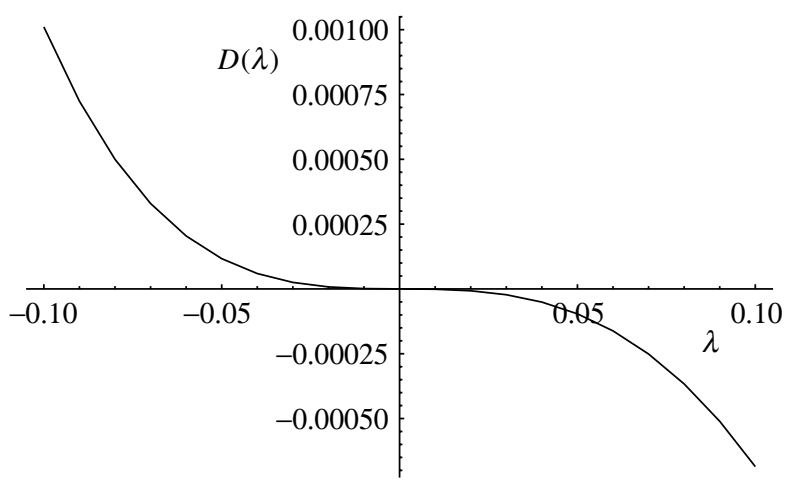

Figure 2. $D(\lambda)$ versus $\lambda$ along the real axis for $\omega=2+\sqrt{ } 5, \psi=\tan ^{-1}(2), \rho=1 / \sqrt{ } 5$.

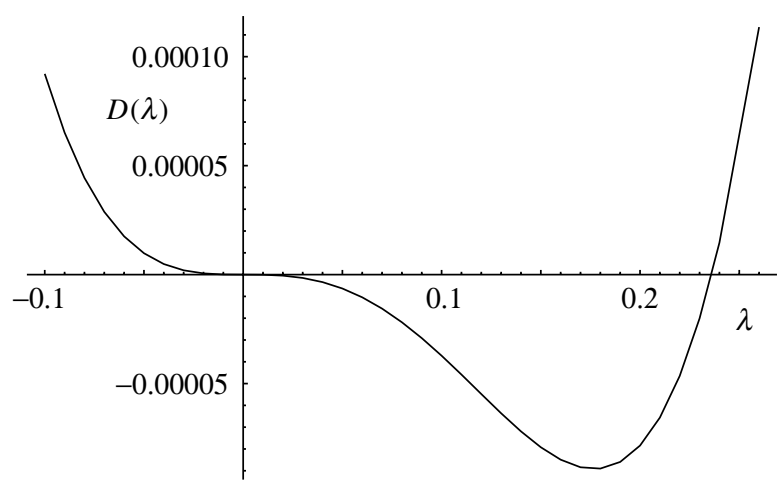

Figure 3. $D(\lambda)$ versus $\lambda$ along the real axis for $\omega=0.03, \psi=\pi / 2, \rho=1$.

integrator is only second-order accurate. Reducing the size of $\Delta x$ further leads to smaller values for the first and second derivatives, suggesting convergence to the exact result.

When $\psi=\pi / 2$ and $\omega=0$, equation (1.4) reduces to the nonlinear Schrödinger equation. According to Weinstein (1985), the eigenvalue $\lambda=0$ is four-fold. In Kapitula (1998), it was demonstrated that for generic and small enough $\left(\left|\varepsilon_{1}\right|,\left|\varepsilon_{2}\right|\right)$ for $\psi=\pi / 2+\varepsilon_{1}, \omega=\varepsilon_{2}$, the quadruple eigenvalue splits into $\lambda_{1}<0$, a double eigenvalue $\lambda_{2}=0$ and $\lambda_{3}>0$.

Our calculations give a numerical evidence of the existence of the triple eigenvalue $\lambda=0$ for $\psi=\pi / 2$ and $\omega>0$ (see figure 3 ), but we have no proof for this fact so far.

Table 2 suggests the existence of the integer-valued eigenvalue $\lambda=15$ and numerics show that the corresponding eigenfunction is even. In fact, this can be demonstrated analytically.

Proposition 5.1. Suppose that $\omega=\frac{3}{2} \tan \psi>0$. Then problem (2.6) has an eigenvalue

$$
\lambda=\frac{3}{\rho \cos \psi} .
$$

Proof. Note that, for $\lambda \in \mathbb{R}$, the eigenvalue problem (2.6) can be written as

$$
\rho \lambda A=\mathrm{e}^{-\mathrm{i} \psi}\left\{A_{x x}-(1+\mathrm{i} \omega)^{2} A+(1+\mathrm{i} \omega)(2+\mathrm{i} \omega)\left(2|\hat{A}|^{2} A+\hat{A}^{2} \bar{A}\right)\right\} .
$$


Table 3. Computed unstable exponent along the neutral curve

\begin{tabular}{lcccc}
\hline$\alpha$ & $\rho$ & $\psi$ & $\omega$ & $\lambda$ \\
\hline 1.0973 & 0.069720 & -1.219908 & -3.399210 & 0.650261 \\
1.09 & 0.072627 & -1.306955 & -4.228913 & 0.896550 \\
1.08 & 0.074454 & -1.356560 & -4.946956 & 1.120561 \\
1.07 & 0.075865 & -1.392846 & -5.668508 & 1.356206 \\
1.06 & 0.077080 & -1.422892 & -6.462923 & 1.628036 \\
1.05 & 0.078173 & -1.449108 & -7.377601 & 1.957210 \\
1.04 & 0.079180 & -1.472663 & -8.466342 & 2.371709 \\
1.03 & 0.080122 & -1.494230 & -9.803213 & 2.914473 \\
1.02055 & 0.080965 & -1.513175 & -11.39539 & 3.609562 \\
1.01 & 0.081861 & -1.532987 & -13.74404 & 4.731599 \\
1.00 & 0.082672 & -1.550681 & -16.86346 & 6.400421 \\
0.988 & 0.083603 & -1.567483 & -21.51673 & 9.270247 \\
\hline
\end{tabular}

Take the ansatz

$$
A(x)=A_{0}(\cosh x)^{-\sigma}, \quad A_{0} \in \mathbb{C}, \quad \text { with } \sigma=r+\mathrm{i} \nu, \quad r>0,
$$

suggested to the authors by A. Mielke. Then substitution leads to $\nu=\omega$ and $r=2 \omega \cot \psi-1$. From the complex equation

$$
(1+\mathrm{i} \omega)(2+\mathrm{i} \omega)\left(2+\mathrm{e}^{-2 \mathrm{i} \arg A_{0}}\right)=(-2 \omega \cot \psi+1-\mathrm{i} \omega)(-2 \omega \cot \psi-\mathrm{i} \omega),
$$

we get two branches. The first one, given by $\omega=\tan \psi, A_{0}=\mathrm{i}$, corresponds to $\lambda=0$. The second one is given by $\omega=\frac{3}{2} \tan \psi, \arg A_{0}=-\frac{1}{2} \arccos \left(\left(1-\omega^{2}\right) /\left(1+\omega^{2}\right)\right)$ and corresponds to the eigenvalue $\lambda=3 / \rho \cos \psi$.

For the case corresponding to figure $1, \rho=\cos \psi=1 / \sqrt{ } 5$ and $\omega=3$, resulting in an eigenvalue $\lambda=15$.

From perturbation theory, it follows that in the vicinity of the curve $\omega=\frac{3}{2} \tan \psi$ in the parameter space $(\omega, \psi)$, the eigenvalue $\lambda(\omega, \psi)$ will remain real, but the corresponding eigenvalue or eigenfunction does not appear to be given by a an explicit analytic expression.

\section{Spanwise modulation of plane Poiseuille flow}

In this section we consider values of $\omega, \rho$ and $\psi$ that are associated with the spanwise modulation equation (1.2), and vary along the neutral curve. Note that the streamwise modulation equation (1.1) is only valid near the nose of the neutral curve, whereas the spanwise modulation equation (1.2) is valid all along the neutral curve. Parametrizing the neutral curve for plane Poiseuille flow by values of $\alpha$, the first column of table 3 shows values of $\alpha$ near the nose of the neutral curve (the nose corresponds to $\alpha=1.02055$ in the table). For each point, Afendikov \& Mielke (2001a) have computed the corresponding values of $\rho, \omega$ and $\psi$ that appear in equation (1.4), and these values are shown in the second to fourth columns of table 3. The fifth column is the computed unstable exponent. The growth rate is smallest near the point on the neutral curve of maximum $\alpha$ and increases monotonically as 
Table 4. Effect of $L_{\infty}$ and $\Delta x$ on the computed exponent

\begin{tabular}{ccc} 
& $\lambda, L_{\infty}=15$ & $\lambda, L_{\infty}=30$ \\
$\alpha$ & $\Delta x=0.003$ & $\Delta x=0.0015$ \\
\hline 1.0973 & 0.6502618660 & 0.6502426100 \\
1.09 & 0.8965501700 & 0.8965073601 \\
1.08 & 1.1205615612 & 1.1204845291 \\
1.07 & 1.3562063959 & 1.3560768112 \\
1.06 & 1.6280362498 & 1.6278205750 \\
1.05 & 1.9572104258 & 1.9568470766 \\
1.04 & 2.3717099536 & 2.3710807722 \\
1.03 & 2.9144738595 & 2.9133382230 \\
1.02055 & 3.6095625701 & 3.6074718707 \\
1.01 & 4.7315997831 & 4.7271176831 \\
1.00 & 6.4004213707 & 6.3901268469 \\
0.988 & 9.2702475481 & 9.2427232656 \\
\hline
\end{tabular}

$\alpha$ decreases. In table 4 the sensitivity to changes in $L_{\infty}$ and $\Delta x$ of the value of the unstable eigenvalue is shown. The most difficult region to compute is for $\alpha$ below the nose. In this region the magnitude of $\omega$ is quite large (see column 4 of table 3 ), and therefore the basic state is very oscillatory (see the expression for $\hat{\boldsymbol{q}}$ in (2.3)), and therefore significantly more points are required in the integration. For all computed parameters along the neutral curve there is at least one unstable eigenvalue.

\section{Concluding remarks}

The numerical results show that the HS pulse, with parameter values relevant to the neutral curve and the spanwise modulation of plane Poiseuille flow, is unstable. However, the results on the existence of pulse solutions to three-dimensional NavierStokes problem were obtained using the cGL equation as a scaled problem and are therefore only valid for very-small-amplitude states. It is known that the largeamplitude branches of periodic travelling states bifurcating from plane Poiseuille flow have a turning point and stabilize at large amplitude and low Reynolds number (low relative to the critical value of $R=5772.22$ ). Therefore, it may be that the singlepulse and the nearby multi-pulse solitary waves, which are localized in the spanwise direction, may exist as branches extending to large amplitude, may also stabilize and play a role in the development of spatio-temporal complexity in three-dimensional plane channel flow.

This work was partly supported by a grant awarded by the London Mathematical Society Scheme 5 program, which funded a visit of A.L.A. to the University of Surrey in April 2000, by RFBR 99-01-01157 and by Volkswagen-Stiftung under Grant VW/71016. The authors are thankful to Professor A. Mielke for useful remarks and stimulating discussions.

\section{References}

Afendikov, A. L. \& Mielke, A. 1995 Bifurcations of Poiseuille flow between parallel plates: three-dimensional solutions with large spanwise wavelength. Arch. Ration. Mech. Analysis 129, 101-127.

Proc. R. Soc. Lond. A (2001) 
Afendikov, A. L. \& Mielke, A. 1999 Bifurcation of homoclinic orbits to a saddle-focus in reversible systems with $S O(2)$-symmetry. J. Diff. Eqns 159, 370-402.

Afendikov, A. L. \& Mielke, A. 2001a Multi-pulse solution to the Navier-Stokes problem between parallel plates. Z. Angew. Math. Phys. (In the press.)

Afendikov, A. L. \& Mielke, A. $2001 b$ Nonlocal modulation equations for viscous fluid layer problems and persistence of spatially localized perturbations. Z. Angew. Math. Mech. (In the press.)

Alexander, J. C., Gardner, R. \& Jones, C. K. R. T. 1990 A topological invariant arising in the stability analysis of traveling waves. J. Reine Angew. Math. 410, 167-212.

Allen, L. \& Bridges, T. J. 2000 Numerical exterior algebra and the compound matrix method. University of Surrey. Preprint.

Beyn, W.-J. \& Lorenz, J. 1999 Stability of traveling waves: dichotomies and eigenvalue conditions on finite intervals. Numer. Funct. Analysis Optim. 20, 201-244.

Bridges, T. J. 1999 The Orr-Sommerfeld equation on a manifold. Proc. R. Soc. Lond. A 455 , 3019-3040.

Bridges, T. J. \& Derks, G. 1999 Hodge duality and the Evans function. Phys. Lett. A 251, 363-372.

Davey, A. 1979 On the removal of the singularities from the Riccati method. J. Comp. Phys. 30, 137-144.

Davey, A., Hocking, L. M. \& Stewartson, K. 1974 On the nonlinear evolution of three-dimensional disturbances in plane Poiseuille flow. J. Fluid Mech. 63, 529-536.

Drazin, P. G. \& Reid, W. H. 1981 Hydrodynamic stability. Cambridge University Press.

Evans, J. W. 1975 Nerve axon equations. IV. The stable and unstable impulse. Indiana Univ. Math. J. 24, 1169-1190.

Evans, J. W. \& Feroe, J. 1977 Local stability theory of the nerve impulse. Math. Biosci. 37, 23-50.

Gardner, R. \& Zumbrun, K. 1998 The gap lemma and geometric criteria for instability of shock profiles. Commun. Pure Appl. Math. 51, 797-855.

Hocking, L. M. \& Stewartson, K. 1972 On the nonlinear response of a marginally unstable plane parallel flow to a two-dimensional disturbance. Proc. R. Soc. Lond. A 326, 289-313.

Kapitula, T. 1998 Stability criterion for bright solitary waves of the perturbed cubic-quintic Schrödinger equation. Physica D 116, 95-120.

Kapitula, T. \& Sandstede, B. 1998 Stability for bright solitary wave solutions to perturbed nonlinear Schrödinger equations. Physica D 124, 58-103.

Mielke, A. 2000 The Ginzburg-Landau equation and its role as a modulation equation. In Handbook for dynamical systems, vol. III. Towards applications (ed. B. Fiedler, G. Iooss, N. Kopell \& F. Takens). Springer.

Ng, B. S. \& Reid, W. H. 1979 An initial-value method for eigenvalue problems using compound matrices. J. Comp. Phys. 30, 125-136.

Ng, B. S. \& Reid, W. H. 1985 The compound matrix method for ordinary differential systems. J. Comp. Phys. 58, 209-228.

Pego, R. L., Smereka, P. \& Weinstein, M. I. 1993 Oscillatory instability of traveling waves for a KdV-Burgers equation. Physica D 67, 45-65.

Stewartson, K. \& Stuart, J. T. 1971 A nonlinear instability theory for a wave system in plane Poiseuille flow. J. Fluid Mech. 48, 529-545.

Swinton, J. \& Elgin, J. 1990 Stability of travelling pulse to a laser equation. Phys. Lett. A 145, 428-433.

Weinstein, M. 1985 Modulation stability of ground states of nonlinear Schrödinger equation. SIAM J. Math. Analysis 16, 472-491. 